\section{True orthodontic intrusion using three-piece intrusion arch for correcting excessive gingival exposure}

\author{
Kavitha Odathurai Marusamy, ${ }^{1}$ \\ Saravanan Ramasamy, ${ }^{2}$ \\ Butchi Raju Akondi, ${ }^{3}$ \\ George Jose Cherackal ${ }^{4}$
}

${ }^{1}$ Department of Preventive Dental

Sciences, Ibn Sina National College for

Medical Studies, Jeddah, Saudi Arabia;

${ }^{2}$ Department of Oral and Maxillofacial

Rehabilitation, Ibn Sina National

College for Medical Studies, Jeddah,

Saudi Arabia; ${ }^{3}$ Department of Clinical

Pharmacy and Pharmacology, Ibn Sina

National College for Medical

Studies, Jeddah, Saudi Arabia;

${ }^{4}$ Annoor Dental College and Hospital

Ernakulam, India

\begin{abstract}
The combination of proclined upper anteriors with high gingival exposure is challenging for the orthodontist. Correction of proclined upper anteriors sometimes leads to deepening of the bite and loss of posterior anchorage resulting in worsening of gingival exposure. Routinely correction of high gingival exposure was done prior to space closure resulting in increased treatment duration. However, application of sound biomechanical strategies can help us overcome these challenges without compromising treatment time. This presentation will describe the meticulous orthodontic biomechanics using a 3-piece intrusion arch to simultaneously correct excessive gingival exposure as well as accomplish space closure. The patient presented with Class I malocclusion with proclined upper anterior teeth, crowding in upper and lower arches and an excessive gingival exposure. Fixed orthodontic therapy was initiated with first premolar extractions and the primary strategies after correcting of the crowding was the effective use of a 3-piece intrusion arch for simultaneous intrusion and retraction of proclined anterior teeth. Biomechanics strategies utilizing the 3 piece intrusion arch effectively aided in closure of spaces, correction of high gingival exposure, intrusion of the upper anteriors and controlling posterior anchorage. All desired treatment outcomes were achieved without prolonging treatment time. Proper biomechanics strate-
\end{abstract}

gies can effectively bring about true intrusion of the upper anteriors as well as correct the upper incisor proclination without prolonging treatment time. The use of threepiece intrusion arch to achieve orthodontic correction assures the attainment of predictable treatment results. Loss of anchorage is seldom observed because of the tip back moment on the posterior teeth. Another advantage of intrusion mechanics is the control of the vertical dimension.

\section{Introduction}

Though the correction of excessive gingival exposure is routinely achieved through orthodontic treatment, the need for careful diagnosis and sequential treatment plan is necessary for good results. ${ }^{1}$ Although, the main demand of laypeople is to have perfectly straightened white teeth, they have become more interested in having Hollywood smile in which a full set of wellaligned white teeth that follow the arc of the lower lip, with minimal gingival show. ${ }^{2}$ Because gummy smile is frequently associated with deep overbite, intrusion of upper anterior teeth is one of the main strategies used for its orthodontic treatment. ${ }^{3}$

The combination of proclined upper anteriors with high gingival exposure is challenging for the orthodontist. Correction of proclined upper anteriors sometimes leads to deepening of the bite and loss of posterior anchorage resulting in worsening of gingival exposure. Patients presenting with a convex profile will not benefit from the use of extrusive mechanics because of their facial convexity will worsen, the occlusal plane will steepen, and the lower facial height will increase. The stability of such changes is questionable, especially if patients do not experience adequate growth. For treatment of gummy smile along with deep overbite, in cases of increased facial height, absolute intrusion of the maxillary incisors is required, rather than extrusion of posterior teeth. ${ }^{4-6}$

Conventionally, correction of high gingival exposure was done prior to space closure resulting in increased treatment duration. Several appliances and techniques have been recommended to control the vertical reactive movement of posterior teeth while intruding anterior teeth which includes high-pull headgear, J-hooks headgear, tips back in the arch wire, trans-palatal arch (TPA), segmented arches with basal intrusion arch, and CTA arch wire. Unfortunately, they are either complicated or rely on excellent patient's cooperation, which is highly unreliable. ${ }^{1,7-9}$
Correspondence: Butchi Raju Akondi, Department of Clinical Pharmacy and Pharmacology, Ibn Sina National College for Medical Studies, Jeddah, Saudi Arabia.

E-mail: drraju2020@gmail.com

Key words: Orthodontic intrusion; Threepiece intrusion arch; Gingival exposure.

Conflict of interest: the authors declare no potential conflict of interest.

Received for publication: 28 May 2018 Revision received: 4 September 2018. Accepted for publication: 17 September 2018

This work is licensed under a Creative Commons Attribution NonCommercial 4.0 License (CC BY-NC 4.0).

(C) Copyright B.R. Akondi et al., 2018

Licensee PAGEPress, Italy

Surgical Techniques Development 2018; 8:7762 doi:10.4081/std.2018.7762

\section{Methods to intrude the anterior teeth}

In the past, intrusion of anterior teeth was being done by the injudicious use of continuous arch wires, which led to the extrusion of posterior teeth. Extrusion of the posterior teeth leads to downward tipping of the palatal plane and steepening of the mandibular plane would be noted..$^{10}$

Utility arches are used to correct the deep overbite in the bio progressive technique. 016" X.016" blue Elgiloy arch wire is placed into the brackets of the four incisors, bypassing the canines and premolars. Tip back bend is incorporated mesial to the first molars and are typically 450 to the horizontal plane. The incisors experience the intrusive force with a count clock wise moment, which tends to flare them. Controlling the vertical dimension is challenging, and high pull head gear may be used when necessary. ${ }^{11}$

In Begg's technique, bite opening is achieved bilateral tip back bend given anterior to the permanent molars using Australian 0.016" stainless steel wire. Simultaneous flaring of the incisors and relative intrusion occur when the horizontal distance from the bracket to the center of resistance increases. The molars experience considerable tip back moments and extrusion which can be controlled by incorporating posterior expansion in the wire. ${ }^{12}$

A 2X4 appliance, consisting of an archwire connecting two molars with four incisors, is often used in the mixed dentition. Tip back bends are placed mesial to the 
tube of the molars and the archwire is tied directly into the brackets of the anterior teeth. Intrusion of the anterior teeth occurs with variable amounts of flaring, depending upon the axial inclination of the anteriors. Vertical dimension can be maintained with the use of a high pull headgear. ${ }^{13}$

Burrstone indicates the segmented arch technique to follow incisor intrusion is very successful in preventing extrusion of the posterior teeth. Basically, this system consists of the posterior anchorage unit, an anterior segment and intrusive arch spring. The posterior anchorage unit includes a 0.018 " x 0.025 " stainless steel archwire stabilizing premolars and molars in the right and left sides which are united by trans palatal arch. ${ }^{14}$

The use of J hook and high pull headgear associated with continuous arch wire has been advocated for the treatment of deepbite. The amount of force delivered to the anterior teeth are substantial and may result in severe resorption of the roots of the anterior teeth. The forces delivered by the headgear are also intermittent and the correction of deep overbite is less efficient in intermittent forces than the use of continuous light forces. ${ }^{15}$

Gummy smile, which is mainly due to maxillary dentoalveolar over growth, can be treated effectively with intrusion using anterior TADs, particularly in case of divergent face. ${ }^{16}$

However, application of sound biomechanical strategies can help us overcome these challenges without compromising treatment time. In order to develop a predictable and desirable force system to intrude flared incisors, in 1997 Bhavna Shroff introduced three piece intrusion arch which has precise determination of the point of application of the intrusive force as well as its direction, which are critical in the simultaneous intrusion and retraction of the anterior teeth. ${ }^{1}$ It has proper vertical dimension control and the design of the appliance allows the clinician to deliver a well-controlled force system with minimal chairside adjustments. This presentation will describe the meticulous orthodontic biomechanics using a 3-piece intrusion arch to simultaneously correct excessive gingival exposure as well as accomplish space closure.

\section{Case Report}

A 15-year-old female who came to our hospital having chief complaints of over exposure of upper front teeth and unable to approximate lips. There were no significant medical and dental history and no history of deleterious habits in childhood. Extra oral examination (Figure 1) showed convex profile, dolicocephalic head, leptoprosopic facial form, increased lower facial height, high mandibular plane angle. On soft tissue examination she had incompetent and everted upper lips, acute nasolabial angle, increased incisal display at rest and smile, increased interlabial gap. Intraoral examination (Figure 1) showed Class I molar and canine on both sides, increased overjet of 5 $\mathrm{mm}$, increased overbite of $6 \mathrm{~mm}$, mild crowding in the lower anterior segment, buccal pit dental caries present in 36 and 46. The pre-operative orthopantogram shows presence of all permanent teeth and lateral cephalogram findings (Table 1) reveals prognathic maxilla, orthognathic mandible,
Class II skeletal base, high mandibular plane angle, ANB $5^{0}\left(\mathrm{SNA} 85^{\circ}, \mathrm{SNB} 80 .^{\circ}\right)$, Mandibular plane angle (FMA 220). Moderate crowding in lower arch, No TMJ Signs and Symptoms.

\section{Treatment objectives}

On maxilla: Relieve crowding, retract incisors to ideal axial inclination, Correct crowding in the anterior segment and achieve ideal overjet, intrude upper anteriors, Maintaining the intermolar width. On Mandible: Relieve crowding. Correct the Curve of Spee. Maintain Class I molar and canine relation on both sides. Mildly intrude the lower anteriors to correct deep bite. Maintaining the intermolar and inter-canine

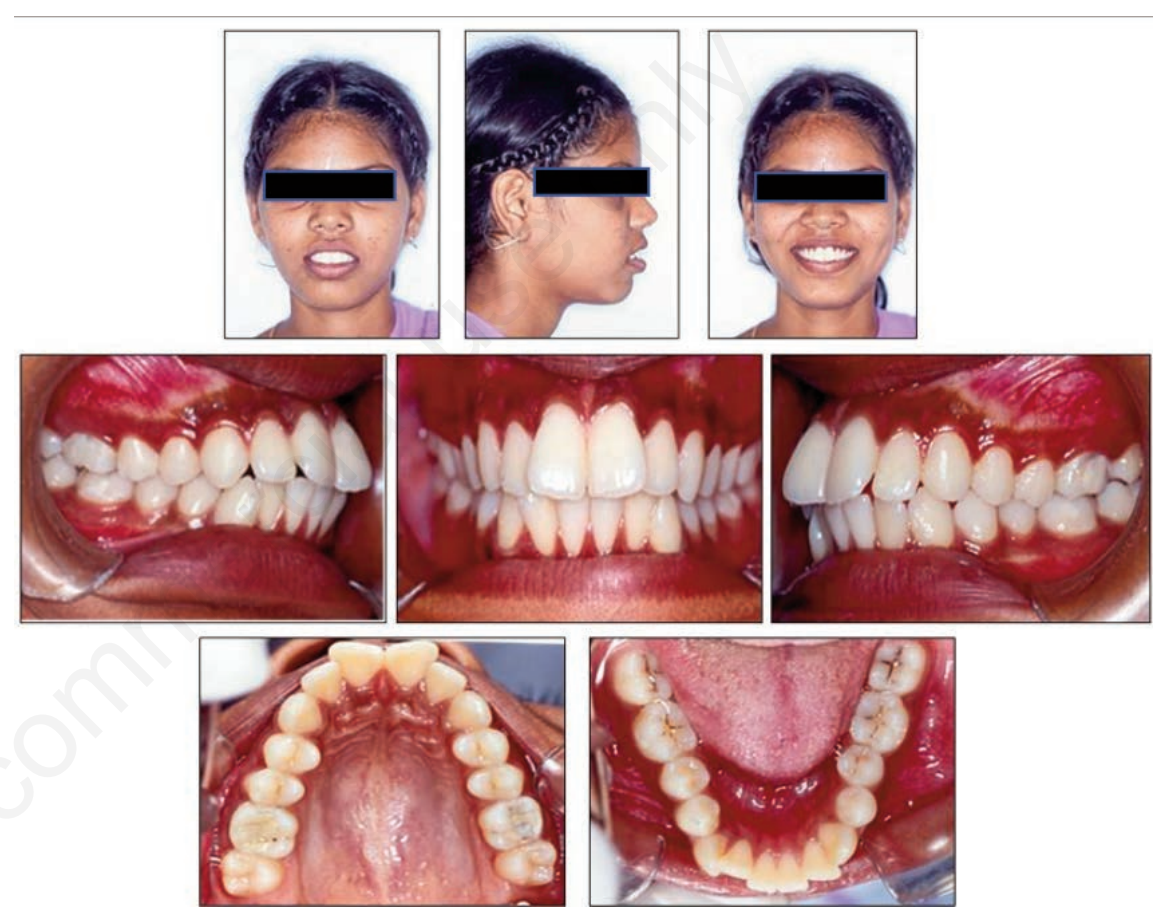

Figure 1. Pre-treatment extra oral and intra oral pictures.

Table 1. The pre-operative orthopantogram shows presence of all permanent teeth and lateral cephalogram findings.

\begin{tabular}{lccc}
\hline AREA & MEASUREMIENT & PRETREATIMINT & POST REATMIENI \\
Max. to Cranial base & SNA & $85^{\circ}$ & $85^{\circ}$ \\
Mand. to Cranial base & SNB & $80^{\circ}$ & $80^{\circ}$ \\
\hline Maxillomandibular & ANB & $5^{\circ}$ & $5^{\circ}$ \\
& Sn to Go-Gn & $33^{\circ}$ & $33^{\circ}$ \\
& FMA & $22^{\circ}$ & $22^{\circ}$ \\
Maxillary Dentition & U1 to NA (mm) & $8 \mathrm{~mm}$ & $3 \mathrm{~mm}$ \\
& U1-SN & $117^{\circ}$ & $103^{\circ}$ \\
& $6-6 \mathrm{~mm}$ (casts) & $48 \mathrm{~mm}$ & $48 \mathrm{~mm}$ \\
& $3-3 \mathrm{~mm}$ (casts) & $34 \mathrm{~mm}$ & $35 \mathrm{~m}(+1)$ \\
\hline Mandibular Dentition & L1 to NB (mm) & $9 \mathrm{~mm}$ & $4 \mathrm{~mm}$ \\
& IMPA & $113^{\circ}$ & $97^{\circ}$ \\
& $6-6 \mathrm{~mm}$ (casts) & $43 \mathrm{~mm}$ & $44 \mathrm{~mm}(+1)$ \\
& $3-3 \mathrm{~mm}$ (casts) & $27 \mathrm{~mm}$ & $27 \mathrm{~mm}$ \\
\hline
\end{tabular}


width. On facial aesthetics, improving facial balance, Improve smile aesthetics.

\section{Treatment progress}

Extraction of 14, 24, 34, 44 were done. Bands with welded molar tubes were cemented to Upper and Lower first molars. Brackets were bonded in upper and lower arch and $0.014 \mathrm{NiTi}$ was placed to begin levelling and aligning, followed by 0.016 $\mathrm{NiTi}$ and $0.019 \mathrm{X} 0.025 \mathrm{NiTi}$. Upper and Lower second molars were banded. TPA was constricted accordingly and placed in relation to upper first molars to achieve correction of the overhanging palatal cusps. Levelling and aligning were done with 0.014 ", 0.018 and .016 "X0.022" NiTi wires and followed by 0.017 "X0.025"and 0.019 X.025" NiTi and finally 0.019X.025" stainless steel was placed (Figure 2). After stage one (aligning and levelling) in this high anchorage case, bite deepening happened due to full expression of tip and torque in canine bracket in $19 \times 25$ stainless steel wire despite our attempts to reduce bite deepening by lace back and reverse curve.

\section{Three-piece intrusion arch appli- ance design}

A rigid anterior segment of wire which was made up of 0.021 "X0.025" stainless steel. It was inserted into the anterior brackets. This anterior wire was stepped up distal to canine to prevent any interferences with the brackets on these teeth during intrusion and retraction. This anterior segment was extended 2-3 $\mathrm{mm}$ distal to the center of resistance of the anterior teeth, posterior stabilizing wire was 0.019 "x0.025" Stainless steel (Figure 3). Bilateral tip back bend was given mesial to first molar on posterior segment $17 \times 25$ TMA wire which was assisting to intrude the anteriors. The point of force application for the intrusive force was distal to the center of resistance of anterior teeth. 30 grams of intrusive forces were applied both right and left sides and small distal force was added by giving an elastomeric chain attaching from molar to the anterior segment of the wire. The force system was monitored and altered carefully at each appointment. The axial inclination was assessed regularly and the estimated position of center of resistance was re-evaluated at each visit. Pre-debonding evaluation was done. Debonding done and post treatment records were taken. Retention was established with fixed Lower canine to canine retainer and Begg retainer in upper arch.

\section{Treatment results}

On maxillary dentition: Mesial movement of molars was controlled. Crowding of anterior corrected. Proclination and uprighting of incisors to a more favourable position was achieved. The vertical position of molars was maintained, and incisors were intruded relatively to reduce the overbite. Intermolar width was maintained. Mandibular Dentition: Crowding in the anterior segment relieved. Class I molar and canine relation achieved on both sides. The vertical position of molars was maintained. Curve of Spee was levelled. Relative intrusion of lower anteriors achieved. Intermolar width and intercanine width was maintained (Table 2). On facial aesthetics:
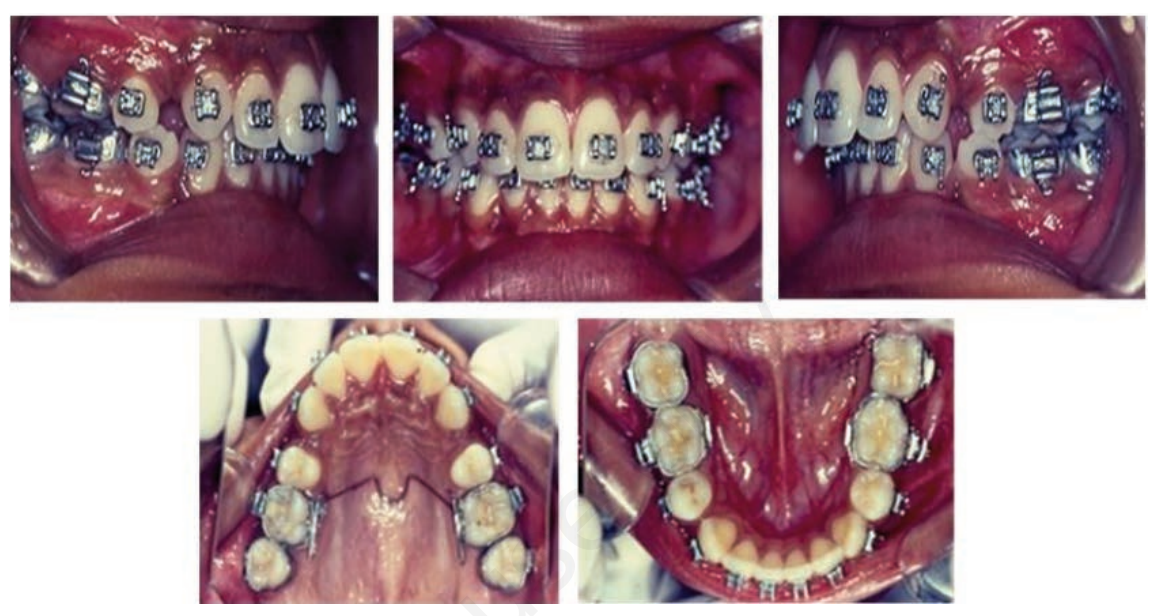

Figure 2. Stage 1 intra oral record.
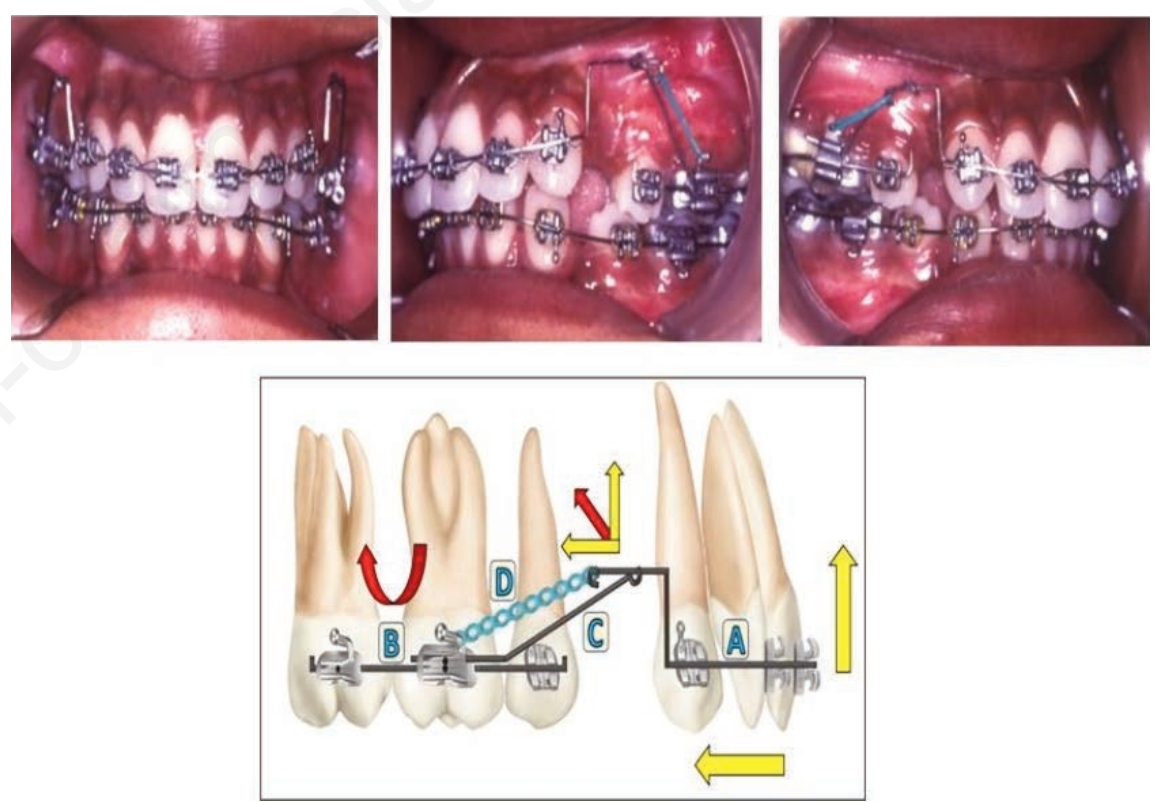

Figure 3. Three-piece intrusion arch.

Table 2. Treatment results.

\begin{tabular}{lcc} 
Soft Tissue Parameters & Pre-Treatment & Post-Treatment \\
S Line to Upper Lip & $7.5 \mathrm{~mm}$ & $+2 \mathrm{~mm}$ \\
S Line to Lower Lip & $6.5 \mathrm{~mm}$ & $3 \mathrm{~mm}$ \\
\hline Nasolabial angle & $86^{\circ}$ & $91^{\circ}$ \\
E Line & $8 \mathrm{~mm}$ & $+1 \mathrm{~mm}$ \\
\hline
\end{tabular}


Improvement of facial profile and smile aesthetics were achieved (Figure 4). Superimposition data reveals vertical position of molars was maintained, absolute intrusion of upper anteriors by $4 \mathrm{~mm}$ and one-degree counter clockwise rotation of mandible (Figure 5).

\section{Discussion}

The smile is the most recognizable signal in the world. Smiles are such an important part of communication that we see them far more clearly than any other expression. Just as a nice smile can act as a powerful communication tool and unpleasant one can have an equally powerful negative impact (as gummy smile). That is why patients seek orthodontic treatment. ${ }^{17}$ Although the correction of gummy smile is routinely achieved through orthodontic treatment, but it needs careful diagnosis and a logically sequenced plan for optimal results. True intrusion as the sole treatment option was questionable in non-growing patients. Amit et al agreed with the treatment of deep bite with intrusion. ${ }^{18}$

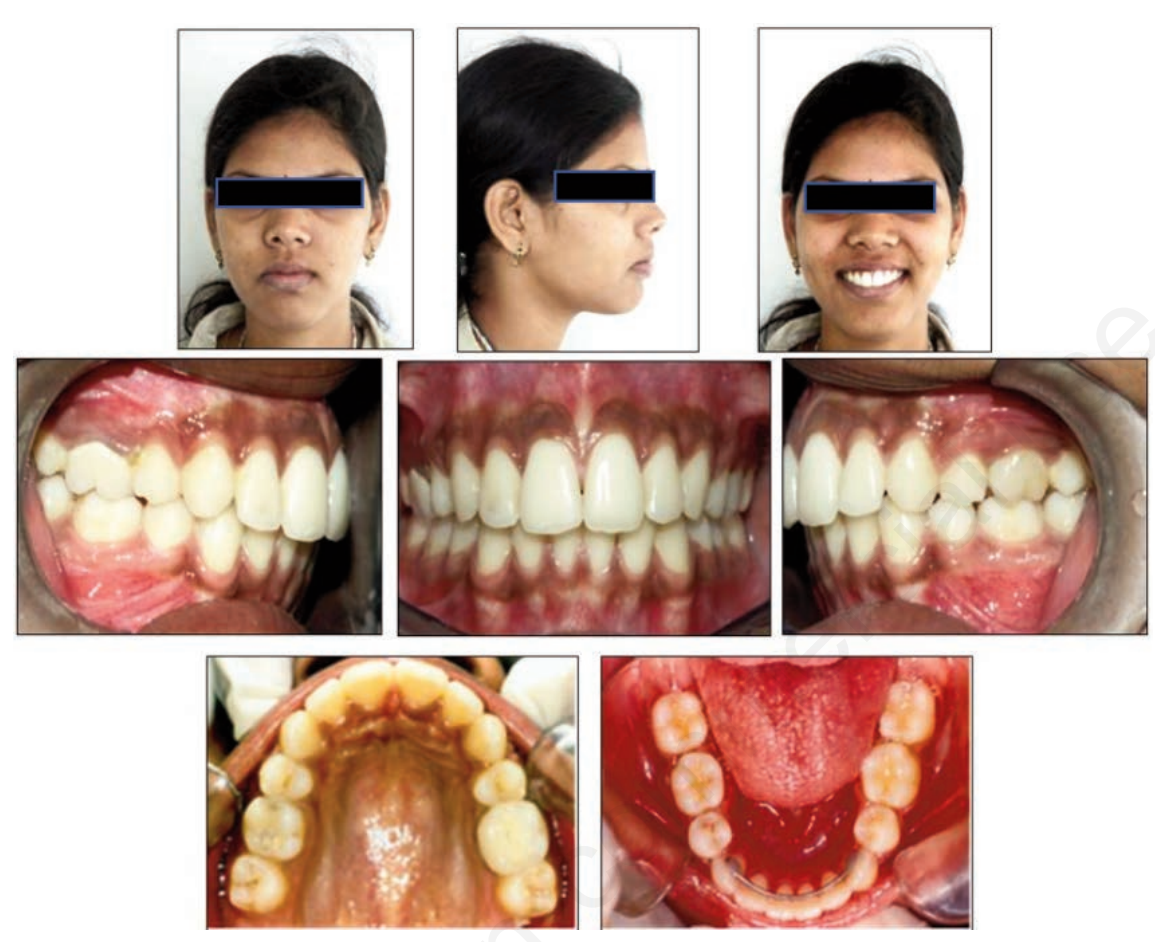

Figure 4. Post-treatment extra oral and intra oral pictures.
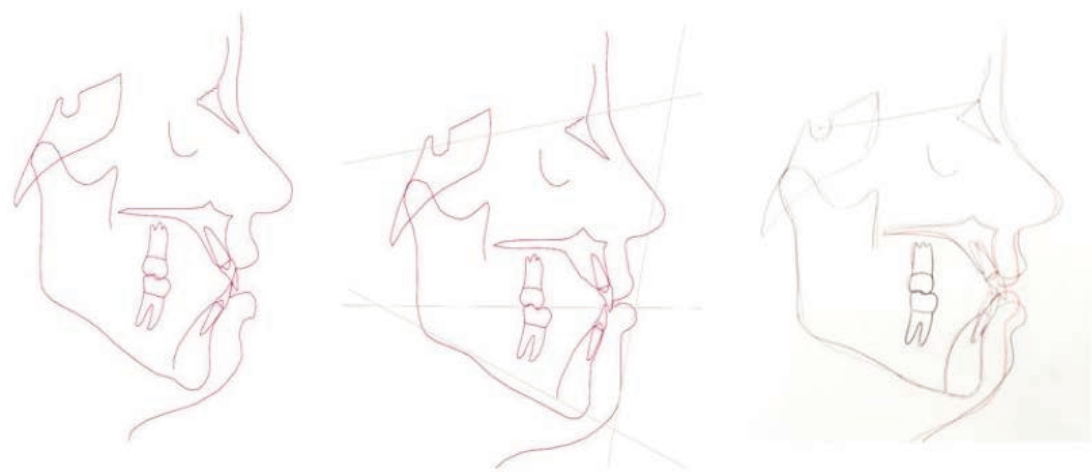

Pre-treatment

Post-treatment

Super-imposition
When crowding and proclination are present, the extraction therapy is necessary, deep overbite correction must be achieved in addition to space closure. Traditionally, the overbite is corrected prior to space closure which consumes more treatment time. However, it is possible to intrude flared incisors and retract these teeth simultaneously using a predictable and precisely analysed force system. ${ }^{19}$ when intrusive force is applied at the center of resistance of the incisors, pure intrusion of teeth is obtained. If intrusion along the long axis of the incisors is indicated, the point of application of the intrusive force can be moved anteriorly and a small distal force will help redirect the intrusive force the long axis of the incisors, thus intruding the incisors along this axis.

In our case report, the patient was having proclined upper anteriors and high gingival exposure.it is mandatory to intrude and retract the upper anteriors to correct the protrusive upper lip, high gingival exposure and control vertical eruption of upper posteriors. Three-piece intrusion arch had given absolute intrusion and retraction of upper anteriors. Applying an intrusive force parallel to the long axis of the incisors and lingual to the center of resistance of the anterior segment of teeth is a more efficient means of achieving simultaneous intrusion and retraction of these teeth. A trans palatal arch, made up of $0.032 \times 0.032$ " stainless steel was placed between the right and left permanent first molars to control molar width as well as axial inclination in the frontal plane. ${ }^{14}$ The intrusion force was added lingual to the center of resistance of the upper anterior teeth and simultaneous retraction (distal) force was applied. ${ }^{20}$ So clockwise moment was produced around the center of resistance of the anterior segment, the upper anteriors were simultaneously intruded and retracted. Thus, few factors like the amount of upper incisors exposure and gingival smile are considered as critical features in the treatment planning. ${ }^{21}$ Avoiding extrusion of the maxillary molars and levelling dentition by incisors intrusion would allow better control of vertical changes. $^{22}$

Ohnishi et al..$^{23}$ described the correction of significant deep bites using miniimplants (TAD's) as anchorage for the intrusion of the upper anterior segment. Mini-implants were placed in between the roots of maxillary central incisors, Kim et $a l .{ }^{24}$ presented a case report wherein they corrected a Class II, Div. 2 deep bite malocclusions by using a mini-implant (TAD) placed below the anterior nasal spine. $4 \mathrm{~mm}$ of incisor intrusion was achieved in 6 months. In our case along with intrusion,

Figure 5. Lateral cephalometric tracings. 
soft tissues change also played a major role to reduces the incisor visibility. In Posttreatment results upper lip length increased by $3.5 \mathrm{~mm}$ and nasolabial angle was increased by 12 degrees. Ismail and Moss (2002) prospectively compared the $2 \mathrm{D}$ and the $3 \mathrm{D}$ effects on the face of extraction and non-extraction orthodontic treatment in patients with skeletal Class I patterns. They showed, based on cephalometric values, that the nasolabial angle was larger in the extraction group, while the vermilion boarder of the upper lip was forward in comparison to the extraction group at the end of treatment. Differential geometrics and surface shape analysis showed that for the two treatment modalities in the current study, there was a significant difference in the changes in upper lip thickness. The reduction in upper lip thickness in the extraction group was accompanied by a decrease in exposed vermilion..$^{25}$

When we study the clinical strategy of the treatment, thorough knowledge about the clinical situation, diagnosis and treatment plan need to be well developed. Visual treatment objectives exact vertical and anteroposterior position of the incisors and molars need to be designed prior to the treatment. Along with this desired force system should be completely analysed to develop an appropriate appliance design. In high anchorage cases, canines are usually retracted and included in the buccal segments of teeth on each side of the arch to allow space for intrusion. ${ }^{14}$

\section{Conclusions}

The use of three-piece intrusion arch to achieve orthodontic correction assures the attainment of a predictable, reproducible and statically determined force system. Loss of anchorage was not observed because of the tip back moment on the posterior tooth. Before starting the treatment, its mandatory to assess the problems, establish clear objectives of treatment, and develop adequate techniques to achieve the established results.

\section{References}

1. Shroff B, Yoon WM, Lindauer SJ, Burstone CJ. Simultaneous intrusion and retraction using a three-piece base arch. Angle Orthod 1997;67:455$61 ; 462$.

2. Van der Geld P, Oosterveld P, Schols J, Kuijpers-Jagtman AM. Smile line assessment comparing quantitative measurement and visual estimation. Am J Orthod Dentofacial Orthoped 2011;139:174-80.

3. Zachrisson BJ. Esthetic Factors Involved in Anterior Tooth Display and the Smile: Vertical Dimension. J Clin Orthodont 1998;35:432-45.

4. Bae SM, Park HS, Kyngn HM, et al. Clinical application of micro-implant anchorage. J Clin Orthodont 2002;36:298-302.

5. Al-Buraiki H, Sadowsky C, Schneider B. The effectiveness and long-term stability of overbite correction with incisor intrusion mechanics. Orthodont Dentofacial Orthoped 2005;127:47-55.

6. Choi YJ, Chung CJ, Choy K, Kim KH. Absolute anchorages with universal $\mathrm{T}$ loop mechanics for sever deep bite and maxillary anterior protrusion and its 10years stability. Angle Orthodontist 2010;80:771-82.

7. Nanda R, Marzban R, Kuhlberg A. The Connecticut intrusion arch. J Clin Orthodont 1998;35:708-15.

8. Foley TF, Sanddu HS, Athanasopoulos C. Esthetic periodontal considerations in orthodontic treatment- the management of excessive gingival display. $\mathrm{J}$ Canad Dent Assoc 2003;69:368-72.

9. Ravindra N, Sunil K. Current Therapy in Orthodontics, 1st Ed. St. Louis: Mosby Elsevier; 2010.

10. Hubbard G, Nanda RS, Currier G. A cephalometric evaluation of nonextraction cervical headgear treatment in Class II malocclusion. Angle Orthod 1994;64:359-70.

11. Moshe Davidovitch, Joe Rebellato. Two-couple orthodontic appliance systems utility arches: a two-couple intrusion arch. Seminar Orthodontics 1995;1:25-30.

12. Xu TM, Lin JX, Kui H, Huang JF. Biteopening mechanics as applied in the Begg Technique. Br J Orthod 1994;21: 189-95.

13. H. Fiona Mckeown, Jonathan Sandler. The Two by Four Appliance: A Versatile Appliance. Dent Update 2001;28:496500.
14. Burstone CJ. Deep overbite correction by intrusion. Am J Orthod 1977;72:122.

15. Nishant Negi, Kehar Singh Negi. Treatment of a severe class II div I malocclusion using J-hook headgear. J Orthodont Res 2014;2:105-8.

16. Pawankumar D, Tekalea KK. Correction of severe deep bite and gummy smile using mini-screw anchorage: A case report. J World Feder Orthodontists 2015;4:162-7.

17. Durgekar S, Naik V. The ideal smile and its orthodontic implications. World J Orthod 2010;11:211-20.

18. Amit P, Arunfhati P, Rahul B, Tarvlatha S. Deep bite correction with Cetlin's intrusion arch. Orthod Cyber J 2011;3:111-20.

19. Shroff B, Lindauer SJ, Burstone CJ, Leiss JB. Segmented approach to simultaneous intrusion and space closure: biomechanics of the three-piece base arch appliance. Am J Orthod Dentofacial Orthop 1995;107:136-43.

20. Liu D, Bai D, Wang C, et al. Simultaneous intrusion and retraction of the anterior teeth using a three-piece base. Arch 2000;18:168-70.

21. Anjali N, Sarojini J, Mohan K. Three piece intrusion arch simplified - a clinical time and motion study. Jaypee Int $\mathrm{J}$ Orthodont 2009;43:38-49.

22. Graber TM, Vanarsdall RL. Orthodontics: current principles and techniques. 2th ed. St Louis: CV Mosby; 2000.

23. Ohnishi H, Yagi T, Yasuda Y, Takada K. A mini-implant for orthodontic anchorage in deep overbite case. Angle Orthod 2005;75;444-52.

24. Kim TW, Kim H, Lee SJ. Correction of deep overbite and gummy smile by using a mini-implant with a segmented wire in a growing class II Division 2 patient. Am J Orthod Dentofacial Orthop 2006;130:676-85.

25. Ismail SF, Moss JP. The ThreeDimensional Effects of Orthodontic Treatment on the Facial Soft Tissues - a Preliminary Study. $\mathrm{Br}$ Dent J 2002;192:104-8. 